\title{
DATA ACQUISITION IN NON-INVASIVE BRAIN-COMPUTER INTERFACE USING EMOTIV EPOC NEUROHEADSET
}

\author{
Liberios VOKOROKOS, Branislav MADOŠ, Norbert ÁDÁM, Anton BALÁŽ \\ Department of Computers and Informatics, Faculty of Electrical Engineering and Informatics, \\ Technical University of Košice, Letná 9, 04200 Košice, Slovak Republic, tel.: +421 55602 2662, \\ e-mail: \{liberios.vokorokos, branislav.mados, norbert.adam, anton.balaz\}@tuke.sk
}

\begin{abstract}
This paper deals with the issue of the brain-computer interface (BCI) - the human-machine interface (HMI) based on acquisition, analysis and transformation of signals generated by the central nervous system (CNS) as the manifestation of its normal function. Brain-computer interface can be seen as the bridge that is building up direct one-way or two-way communication pathway between the brain and the external technical device. Paper introduces techniques based on non-invasive functional imaging of the brain used for data acquisition in non-invasive brain computer interfaces, and is focused on the technique that is reading neural activity of the brain with use of multi-channel electroencephalograph (EEG). As the part of this paper we are introducing our experience with the low-cost commercially available equipment Emotiv EPOC Neuroheadset based on this technology.
\end{abstract}

Keywords: user interface, Brain Computer Interface, BCI, functional brain imaging, multi-channel electroencephalograph, EEG, Emotiv EPOC Neuroheadset

\section{INTRODUCTION}

Brain-computer interface (BCI) research, as the interdisciplinary field on the borders of biology, medicine and informatics, has attracted a lot of attention of scientific community in recent years.

Brain-computer interface is often evaluated as the potential part of the equipment able to improve life of the significant number of people with different motor or voice communication disabilities or with completely paralyzed voluntary muscles, which are caused by degenerative diseases or accidents.

Brain-computer interfaces have the potential to build up new communication interface for assistive technologies, including wheelchair control, environment control, various smart home assistive applications or control of personal computers and software applications including web browsers, typewriters, computer spellers or computer games [1][2][3].

BCIs are often focused on neuroprosthetics applications that are aimed at repair and restoration of damaged hearing, sight or movement. It can be seen as the part of the narrower class of neuroprosthetics, because of brain-computer interface definition, based on the requisite of the direct connection between neuroprosthesis and the central nervous system (CNS). BCIs can be considered as the part of neurorehabilitation tools because of the neural plasticity of human brain [4].

Crossing the boundaries of medical applications, brain-computer interfaces can be used for smart home applications intended as the enhancements of life quality and have been evaluated as the interfaces that allow control of various equipments including mobile robots, robotic arms, flying drones or as the interfaces that allows driving a car [5].

Attention is paid to the possibility to use it as the new user interface of personal computer and entertainment industry is evaluating the potential of the use in recreation including various forms of computer games and virtual reality applications [6].
Brain-computer interface or mind-machine interface (MMI) or brain-machine interface (BMI) is the humanmachine interface (HMI) that can be defined as the system able to translate subject's intent or thoughts into the technical control signals without the use of the communication channel based on the use of the speech or any other neuromuscular activity. BCI is building direct communication pathway between the human brain and the computer or external technical device in general with aim to build one-way or two-way communication interface.

The key components of brain-computer interface systems are data acquisition hardware based on the monitoring of signals produced as the manifestation of normal activity of the central nervous system and software component that is analysing acquired signals and is extracting features that can be translated into the technical control signals appropriate for use in control of the external technical device.

Brain-computer interfaces are forming three main groups according to the invasivity to the body of users.

Invasive brain-computer interfaces are surgically implanted directly into the brain, and are often based on the use of arrays of microelectrodes implanted into the motor or visual cortex. Invasive brain-computer interfaces are able to provide best temporal and spatial resolution of signals. Partially invasive brain-computer interfaces are locating arrays of microelectrodes inside the skull but not inside the brain. Electrocorticography $(\mathrm{ECoG})$ is the example of this technique.

Non-invasive brain-computer interfaces are based on the functional brain imaging techniques, mostly on multichannel electroencephalography (EEG). Although noninvasive BCIs are providing the lowest accuracy in the signal acquisition, because of the deflection caused by the skull, there is advantage in no modification of the human body and minimal discomfort for the user of the interface. It is possible to implement non-invasive brain-computer interfaces under the most naturalistic conditions of use with minimal need of the wearable hardware and with advantage of wireless connection to the host computer that 
is analyzing and transforming signals or to the technical device that is controlled with use of brain-computer interface.

\section{BRAIN IMAGING TECHNIQUES}

The key component of the BCI is the data acquisition hardware intended for monitoring of the manifestation of normal neural activity of the central nervous system. Data acquisition in non-invasive brain-computer interface is based on the brain imaging and this category includes various techniques that are capable directly or indirectly image the structure (structural brain imaging) and function (functional brain imaging) of the brain.

\subsection{Structural brain imaging}

Structural brain imaging deals with the imaging of the brain structure and there is possibility to use it for diagnostic purpose in the case of intracranial diseases including tumours and in case of various injuries.

Computed Tomography (CT) or Computerized Axial Tomography (CAT) uses series of x-ray images of head taken from various directions and creates cross-sectional images of the brain.

Magnetic Resonance Imaging (MRI) uses magnetic fields and radio waves to acquire data for formation of two- or three-dimensional images of the brain. MRI has advantage in no use of ionizing radiation or radioactive tracers.

\subsection{Functional brain imaging}

Functional imaging of the brain is used for diagnostic purpose in case of metabolic diseases and lesions and it is also suitable group of techniques that can be used for neurological and cognitive psychology and for the creation of brain-computer interfaces.

Positron Emission Tomography (PET), using trace amounts of short-lived radioactive materials, Single Photon Emission Computed Tomography (SPECT), using gama-ray emitting radioisotopes, functional Magnetic Resonance Imaging (fMRI) and Near Infrared Spectroscopic Imaging (NIRSI) are techniques that measure and localise changes in cerebral blood flow, which are related to the neural activity and it is possible to use it for identification of regions of the brain, which are activated when subject is performing particular tasks.

Other imaging techniques, including the magnetoencephalography (MEG) used for both research and clinical purpose, electrocorticography (ECoG) and electroencephalography (EEG) are suitable techniques for recording of changes in electrical currents and magnetic fields, which are produced as the manifestation of the normal function of the brain.

Electroencephalography (EEG) can be defined as the measurement of the electrical activity of the brain, realised by recording of electrical signals scanned by electrodes, which are placed on the scalp.

The result of measurement, which is called electroencephalogram (EEG), represents the set of electrical signals scanned from large groups of neurons. This technique is frequently used in research and development aimed at creation of the BCI, because the process of its use is non-invasive for the user. Another advantage is that the EEG is capable of high temporal resolution with measurement of electrical activity of the brain on the level of milliseconds.

All of described techniques have its limitations and differ in advantages and disadvantages. For example MEG, or EEG, which measure neural activity of the brain with high temporal resolution, are limited in ability of spatial resolution and fMRI with its high capability of the localization of neural activity has disadvantage because of its lower temporal resolution.

\section{EMOTIV EPOC NEUROHEADSET}

In our effort to build novel application of the braincomputer interface we are using low-cost commercially available 14 channel EEG hardware Emotiv EPOC Neuroheadset (Fig. 1), which is used for acquisition of raw data from electrodes that are positioned at AF3, F7, F3, FC5, T7, P7, O1, O2, P8, T8, FC6, F4, F8 and AF4 positions, according to the international 10-20 system. Odd numbers of electrodes are reserved for left hemisphere of the brain; even numbers of electrodes are reserved for right hemisphere of the brain.

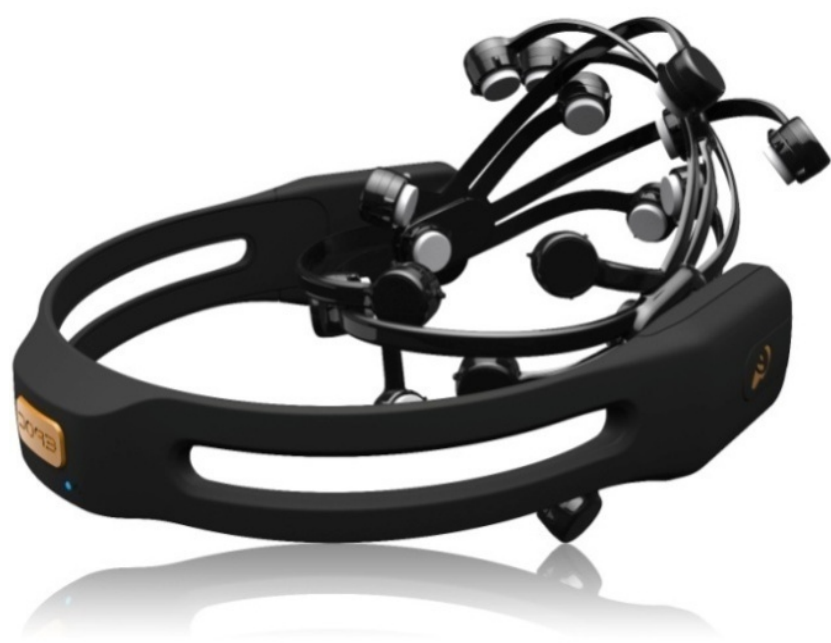

Fig. 1 Low-cost EEG signal acquisition hardware Emotiv EPOC Neuroheadset

Electrodes AF3, AF4, F3, F4, F7 and F8 are used for imaging of neural activity of the lobus frontalis of the subject's brain. Electrodes FC5, FC6, T7 and T8 are scanning the lobus temporalis of the brain. The lobus parietalis is scanned by P7 and P8 electrodes. Neural activity of the lobus occipitalis is scanned with use of the $\mathrm{O} 1$ and $\mathrm{O} 2$ electrodes (Fig. 2).

Two referencing electrodes CMS (on the left side) and DRL (on the right side) are used for reduction of noise in signal. 


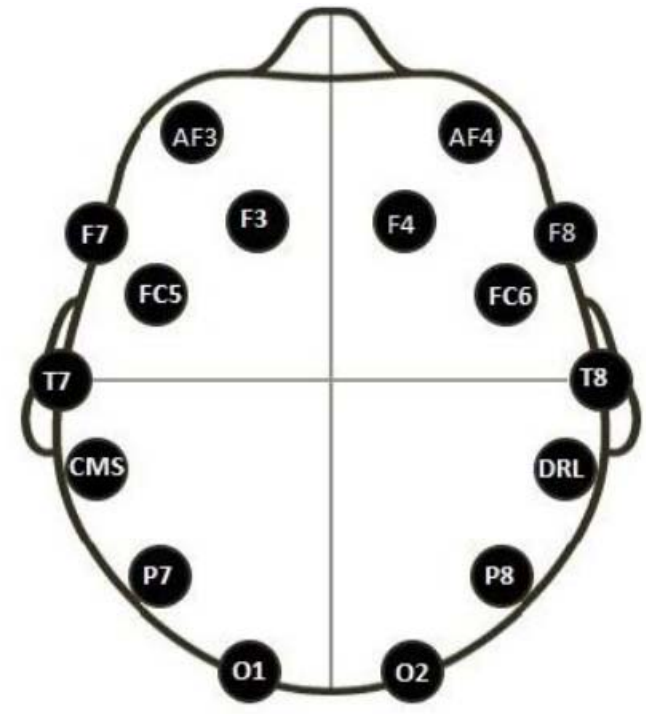

Fig. 2 Electrodes acquiring signals of neural activity of the brain are positioned according to the $10-20$ model

Signals are sampled with $128 \mathrm{~Hz}$ sampling rate and are sent through the wireless connection to the dongle, which is used as a receiver connected to the USB port of personal computer. Host computer is used to perform computationally expensive analysis of acquired data and transformation into the control signals for software applications.

There is possibility to find characteristic patterns in EEG signals that are connected with facial expressions including blink, right or left wink, right or left look, raise or furrow of brow, smile, teeth clench, right or left smirk and laugh and those patterns are, according to tests provided as the part of our research, recognizable in signals from respective electrodes.

For example, look left or right is manifesting mostly in signals from $\mathrm{F} 7$ and $\mathrm{F} 8$ electrodes and also in signals from FC5, O2, P8, FC6 and AF4 as shown in Table 1.

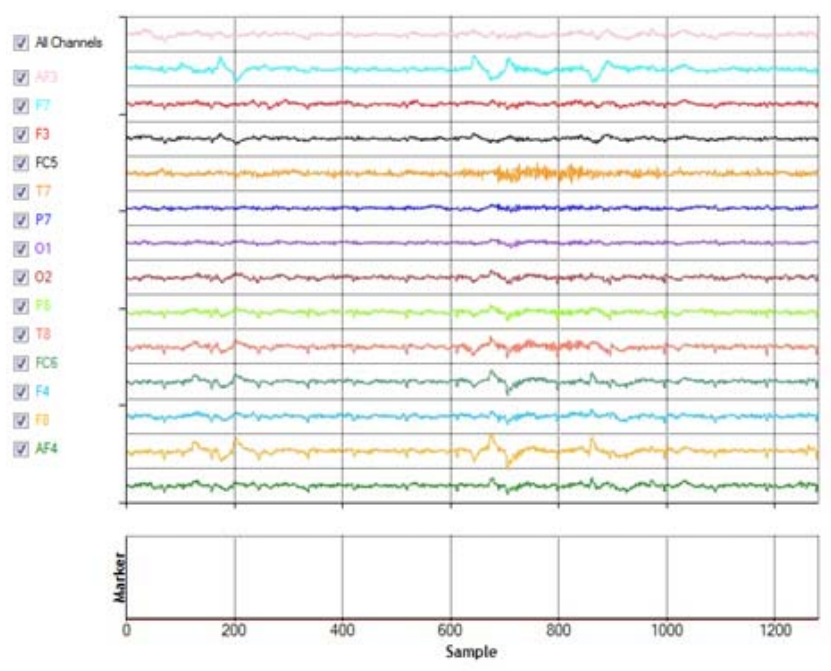

Fig. 3 Characteristic patterns in EEG signals for smile facial expression
Table 1 Recognisability of characteristic patterns of facial expressions in EEG signals

\begin{tabular}{|c|c|c|c|c|c|c|c|c|c|c|c|c|}
\hline & 苞 & $\begin{array}{l}\text { 曾 } \\
\text { 总 }\end{array}$ & 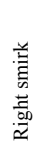 & $\begin{array}{l}\text { 总 } \\
\text { 苛 }\end{array}$ & 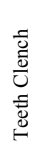 & 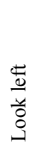 & 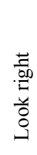 & 盖 & 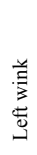 & 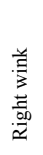 & 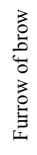 & 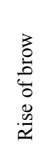 \\
\hline$\overline{A F 3}$ & $\sqrt{ }$ & & $\sqrt{ }$ & $\sqrt{ }$ & $\sqrt{ }$ & & & $\sqrt{ }$ & $\sqrt{ }$ & $\sqrt{ }$ & $\sqrt{ }$ & $\sqrt{ }$ \\
\hline F7 & $\sqrt{ }$ & & $\sqrt{ }$ & $\sqrt{ }$ & $\sqrt{ }$ & $\sqrt{ }$ & $\sqrt{ }$ & $\sqrt{ }$ & $\sqrt{ }$ & $\sqrt{ }$ & $\sqrt{ }$ & $\sqrt{ }$ \\
\hline F3 & & & & $\sqrt{ }$ & $\sqrt{ }$ & & & $\sqrt{ }$ & & $\sqrt{ }$ & $\sqrt{ }$ & $\sqrt{ }$ \\
\hline FC5 & & & & $\sqrt{ }$ & $\sqrt{ }$ & $\sqrt{ }$ & $\sqrt{ }$ & $\sqrt{ }$ & $\sqrt{ }$ & & $\sqrt{ }$ & $\sqrt{ }$ \\
\hline $\mathbf{T} 7$ & $\sqrt{ }$ & $\sqrt{ }$ & & $\sqrt{ }$ & $\sqrt{ }$ & & & & $\sqrt{ }$ & & $\sqrt{ }$ & $\sqrt{ }$ \\
\hline P7 & & $\sqrt{ }$ & & $\sqrt{ }$ & $\sqrt{ }$ & & & & $\sqrt{ }$ & & $\sqrt{ }$ & $\sqrt{ }$ \\
\hline 01 & & & & $\sqrt{ }$ & $\sqrt{ }$ & & & & & & & $\sqrt{ }$ \\
\hline $\mathrm{O} 2$ & $\sqrt{ }$ & & & $\sqrt{ }$ & $\sqrt{ }$ & $\sqrt{ }$ & $\sqrt{ }$ & & & & & $\sqrt{ }$ \\
\hline P8 & $\sqrt{ }$ & & & $\sqrt{ }$ & $\sqrt{ }$ & $\sqrt{ }$ & $\sqrt{ }$ & & & & & $\sqrt{ }$ \\
\hline T8 & $\sqrt{ }$ & & $\sqrt{ }$ & $\sqrt{ }$ & $\sqrt{ }$ & $\sqrt{ }$ & $\sqrt{ }$ & & & & & \\
\hline FC6 & $\sqrt{ }$ & & $\sqrt{ }$ & $\sqrt{ }$ & $\sqrt{ }$ & $\sqrt{ }$ & $\sqrt{ }$ & $\sqrt{ }$ & & $\sqrt{ }$ & $\sqrt{ }$ & $\sqrt{ }$ \\
\hline F4 & $\sqrt{ }$ & & & $\sqrt{ }$ & $\sqrt{ }$ & & & $\sqrt{ }$ & & $\sqrt{ }$ & & $\sqrt{ }$ \\
\hline F8 & $\sqrt{ }$ & & $\sqrt{ }$ & $\sqrt{ }$ & $\sqrt{ }$ & $\sqrt{ }$ & $\sqrt{ }$ & $\sqrt{ }$ & & $\sqrt{ }$ & $\sqrt{ }$ & $\sqrt{ }$ \\
\hline AF4 & $\sqrt{ }$ & & $\sqrt{ }$ & $\sqrt{ }$ & $\sqrt{ }$ & $\sqrt{ }$ & $\sqrt{ }$ & $\sqrt{ }$ & $\sqrt{ }$ & $\sqrt{ }$ & $\sqrt{ }$ & $\sqrt{ }$ \\
\hline
\end{tabular}

Affections of the subject are also manifesting in EEG signals and there is possibility to detect characteristic patterns for engagement, boredom, frustration, meditation, instantaneous excitement or long term excitement, which are connected to the vigilance, alertness, concentration, stimulation, interest, contemplation, expectation or negative feelings.

Intentions of the subject to push, pull, move left, right, up and down and to rotate clockwise, counterclockwise, left, right, forward and backward are detectable. Intents are connected with real life or virtual objects. In case of virtual objects there is possibility to detect intent of the subject to let those objects disappear.

The ability to control external technical device is determined by the capability of proper level of concentration of the subject during the process of signal scanning.

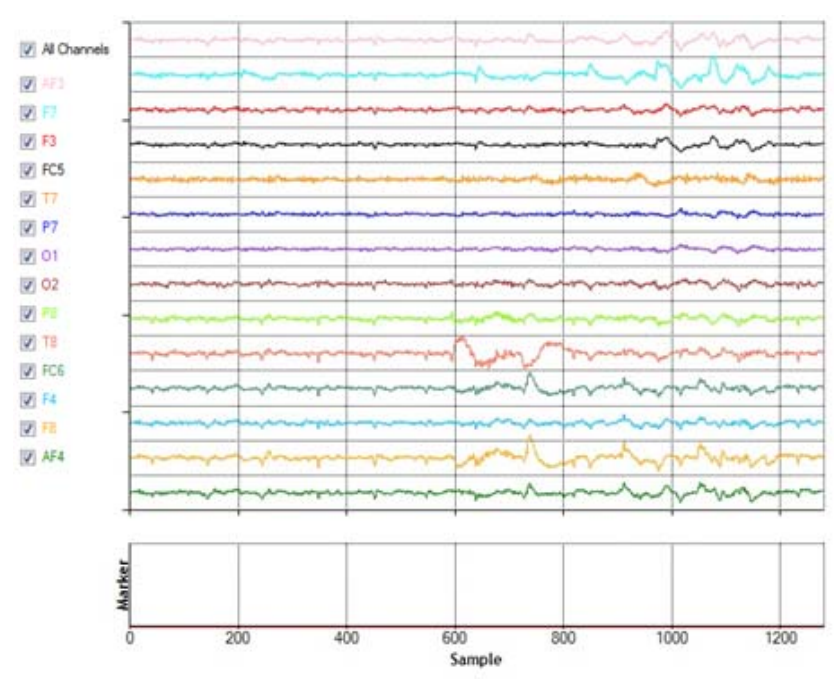

Fig. 4 Characteristic patterns in EEG signals for right smirk facial expression 


\section{CONCLUSIONS}

The paper introduced brain-computer interface as the technology capable of building the communication bridge between central nervous system and external technical device and allows one-way or two-way communication. Techniques of the brain imaging with emphasis on the functional brain imaging techniques and especially the multi-channel electroencephalography (EEG) were introduced and our experience with low-cost EEG hardware Emotiv EPOC Neuroheadset was described.

\section{ACKNOWLEDGMENTS}

This work was supported by the Slovak Research and Development Agency under the contract No. APVV-000810. Project is being solved at the Department of Computers and Informatics, Faculty of Electrical Engineering and Informatics, Technical University of Košice.

\section{REFERENCES}

[1] OBERMAIER, B. - MULLER, G. R. PFURTSCHELLER, G.: Virtual keyboard controlled by spontaneous EEG Activity. IEEE Transactions on neural Systems and Rehabilitation Engineering, Vol. 11, No. 4, December 2003, pp. 422-426.

[2] BIANCHI, L. - QUITADAMO, L. R. GARREFFA, G. - CARDARILI, G. C. MARCIANI, M. G.: Performance Evaluation and Optimization of Brain Computer Interface Systems in a Copy Spelling Task. IEEE Transs on neural Systems and Rehab Engineering, Vol. 15, No. 2, June 2007, pp. 207-216.

[3] LAURENTIS, K. J. - ARBEL, Y. - DUBEY, R. DONCHIN, E.: Implementation of a P-300 brain computer interface for the control of a wheelchair mounted robotic arm system. Published by ASME in the Proceedings of the ASME 2008 Summer Bioengineering Conference (SBC2008), June 25-29, pp. 1-2 (2008).

[4] CULPEPPER, B. J. - KELLER, R. M.: Enabling computer decisions based on EEG Input. IEEE Transactions on neural Systems and Rehabilitation Engineering, Vol. 11, No. 4, December 2003, pp. 354-360.

[5] WOLPAW, J. R. - McFARLAND, D. J.: Control of a twodimensional movement signal by a noninvasive braincomputer interface in humans, Published by Proceedings of the National Academy of Sciences of the United States of America, December 21, 2004, Vol. 101, No. 51.

[6] L'ECUYER, A. - LOTTE, F. - REILly, R. LEEB, R. - HIROSE, M. - SLATER, M.: Braincomputer interfaces, virtual reality and videogames. IEEE Computer, 41(10):66-72, 2008.

Received January 10, 2012, accepted March 16, 2012

\section{BIOGRAPHIES}

Liberios Vokorokos (prof., Ing., PhD.) was born on 17.11.1966 in Greece. In 1991 he graduated (MSc.) with honours at the Department of Computers and Informatics of the Faculty of Electrical Engineering and Informatics at Technical University in Košice. He defended his $\mathrm{PhD}$. in the field of programming device and systems in 2000; his thesis title was "Diagnosis of compound systems using the Data Flow applications". He was appointed professor for Computers Science and Informatics in 2005. Since 1995 he is working as an educationist at the Department of Computers and Informatics. His scientific research is focusing on parallel computers of the Data Flow type. In addition to this, he also investigates the questions related to the diagnostics of complex systems. Currently he is dean of the Faculty of Electrical Engineering and Informatics at the Technical University of Košice. His other professional interests include the membership on the Advisory Committee for Informatization at the faculty and Advisory Board for the Development and Informatization at Technical University of Košice.

Branislav Madoš (Ing., PhD.) was born on 20.5. 1976, in Trebišov, Slovakia. In 2006 he graduated (MSc.) with distinction at the Department of Computers and Informatics at the Faculty of Electrical Engineering and Informatics of the Technical University of Košice. He defended his $\mathrm{PhD}$. in the field of Computers and computer systems in 2009; his thesis title was "Specialized architecture of data flow computer". Since 2010 he has been working as a professor assistant at the Department of Computers and Informatics. His scientific research is focused on the parallel computer architectures and architectures of computers with data driven computational model.

Norbert Ádám (Ing., PhD.) was born on 30.8.1980. In 2003 he graduated (MSc.) with distinction at the Department of Computers and Informatics at the Faculty of Electrical Engineering and Informatics of the Technical University of Košice. He defended his PhD. in the field of Computers and computer systems in 2007; his thesis title was "Contribution to simulation of feed-forward neural networks on parallel computer architectures". Since 2006 he is working as a professor assistant at the Department of Computers and Informatics. Since 2008 he is the head of the Computer Architectures and Security Lab. at the Department of Computers and Informatics. His scientific research is focused on the parallel computers architectures.

Anton Baláž (Ing., PhD.) was born in Sobrance, Slovakia, in 1980. He received the master degree in Informatics in 2004 from Faculty of Electrical Engineering and Informatics, Technical University of Košice. In 2008 he received $\mathrm{PhD}$. in area of computer security. Since 2007 he is working as professor assistant at the Technical University of Košice. 